\title{
Nonlocal Mumford-Shah Model for Image Segmentation
}

\author{
Wenqi Lu' ${ }^{1}$ \\ College of Information Engineering, Qingdao University, Qingdao, 266000, China \\ E-mail:ccluxiaogie163.com
}

\section{Weibo $\mathbf{W e i}^{23}$}

College of Information Engineering, Qingdao University, Qingdao, 266000, China

E-mail: njustwwbe163.com

\section{Zhenkuan Pan}

College of Information Engineering, Qingdao University, Qingdao, 266000,China

E-mail: zkpan@qdu. edu. cn

\section{Jinming Duan}

School of Computer Science, University of Nottingham, Nottingham, NG7 2RD,UK

E-mail: duanmujinming(126.com

\section{Guodong Wang}

College of Information Engineering, Qingdao University, Qingdao, 266000,China

E-mail: allen_wgd@163.com

\begin{abstract}
Mumford-Shah (MS) model is a popular model among image segmentation area. A multiphase nonlocal Mumford-Shah (NLMS) model which combines nonlocal operators and original MS model is proposed in this paper to segment multiphase images. In order to segment different patterns simultaneously, multiple region partition strategy which uses $n-1$ level set functions to segment $n$ regions is adopted. Furthermore, corresponding split Bregman algorithm is designed to improve computational efficiency. Finally, numerical experiments are shown to validate the efficiency and effectiveness of the proposed model.
\end{abstract}

CENet2015

12-13 September 2015

Shanghai, China

\footnotetext{
${ }^{1}$ Speaker

${ }^{2}$ Correspongding Author

${ }^{3}$ The work is supported by the National Natural Science Foundation of China(61170106).
} 


\section{Introduction}

Image segmentation is a technology to segment the image into regions with their own characteristics and extracting targets with interest. It is the fundamental problem of image processing and analysis[1,2], computer vision and machine recognition.

According to different information contained in the image, the active contour model which has been widely used in segmentation can be divided into two types: models based on edges and models based on regions. Models based on the edge such as snake model [3], can get ideal results in the process of segmenting images with obvious edges[4]. However, the contours are often not accurate enough in the process of segmenting images with weak edges. To solve the problems, researchers proposed the Mumford-Shah (MS) model based on regions[5]. This model equals original images to piecewise smooth images and minimal contours by minimizing the energy functional. However, due to the inconsistency in the dimension of image and contours, the problem can not be solved. In order to make it computable, Chan and Vese used the level set method to segment the piecewise constant image[6-8], which was the Chan-Vese (CV) model. To segment inhomogeneous objects in the image, Vese and Chan improved CV model by minimizing the two-phase piecewise smooth MS energy[9]. In terms of texture image segmentation, Sandberg and Chan adopted Gabor transform to convert the original texture image into vector images, and then applied CV model to each layer of vector images[10]. However, due to the existence of some redundant and incorrect layer in vector images, it leads to wrong segmentation results. In 2005, Buades et al first proposed nonlocal method[11]. This method can keep the texture well. In 2008, Gilboa and Osher defined a new series of nonlocal operators[12]. Bresson and Chan combined the piecewise smooth Mumford-Shah model with nonlocal operators to segment two-phase texture images[9,13]. Their approaches overcomes the defects [10]. And the approaches get better results.

In this paper, a multiphase nonlocal Mumford-Shah model which combines nonlocal operators and multiple region partition strategy is proposed for image segmentation[13]. The former can cope with texture information in the image, while the latter can uses $n$ - 1 label functions to segment $n$ regions. The split Bregman method is adopted to transform the energy minimization problem into some subproblems and then solve them respectively.

\section{Two-Phase Nonlocal Mumford-Shah Model and Nonlocal Operators}

First, we will review the two-phase nonlocal Mumford-Shah (NLMS) model. For an image $f(x): \Omega \odot R, x \Omega$, the minimization problem of NLMS model is as follows

$$
\min _{u_{1}, u_{2}, \phi \in[0,1]}\left\{E\left(u_{1}, u_{2}, \phi\right)=\alpha_{1} \int_{\Omega}\left(\left(u_{1}-f\right)^{2}+\lambda_{1}\left|\nabla_{N L} u_{1}\right|^{2}\right) \phi d x+\alpha_{2} \int_{\Omega}\left(\left(u_{2}-f\right)^{2}+\lambda_{2}\left|\nabla_{N L} u_{2}\right|^{2}\right)(1-\phi) d x+\gamma \int_{\Omega}|\nabla \phi| d x\right\}
$$

where $u_{1}$ and $u_{2}$ is the gray value inside and outside of the closed contour denoted by label function $\phi$ respectively. $\alpha_{1}, \lambda_{1}, \alpha_{2}, \lambda_{2}$ and $\gamma$ are the penalty parameters balancing these three energy terms. $\quad{ }_{N L} u$ is the gradient operator defined in the nonlocal space. In order to solve (2.1), we need the nonlocal operators.

Based on nonlocal means, Gilboa and Osher systematically defined nonlocal gradient operator, divergence operator and Laplacian etc[12]. Define $u(x): \Omega \quad R$ be the grayscale image which is defined on image space $\Omega$, nonlocal similarity of $x: x \quad \Omega$ and $y: y \quad \Omega$ can be defined as

$$
w(x, y)=\exp \left\{-\frac{G_{\sigma} *(\|u(x+\cdot)-u(y+\cdot)\|)^{2}}{h^{2}}\right\}
$$

where $G_{\sigma}$ is the Gaussian kernel function, $h$ is the thresholding value controlling similarity,$\sigma$ is the standard deviation of Gaussian kernel function. With the definition of nonlocal similarity, at point $x$, nonlocal gradient vector operator is shown as 


$$
\nabla_{N} u(x, y) \triangleq(u(y)-u(x)) \sqrt{w(x, y)}: y \in \Omega
$$

At point ${ }^{x}$, the nonlocal gradient module value is as follows

$$
\left|\nabla_{N \Sigma} u\right|(x)=\sqrt{\int_{\Omega}(u(y)-u(x))^{2} w(x, y) d y}
$$

The nonlocal divergence at point $x$ is

$$
\left(\nabla_{N L} \cdot \vec{p}\right)(x) \triangleq \int_{\Omega}(p(x, y)-p(y, x)) \sqrt{w(x, y)} d y
$$

Then the nonlocal Laplace at point $x$ is

$$
\Delta_{N L} u(x) \triangleq \frac{1}{2} \nabla_{N L} \cdot\left(\nabla_{N L} u(x)\right)=\int_{\Omega}(u(y)-u(x)) w(x, y) d y
$$

Model (2.1) can only segment two-phase images and the optimisation method (i.e. gradient decent flow) used to solve label function $\phi$ is difficult to discretise to solve. Therefore, it is a nontrivial problem proposing multiphase segmentation model.

\section{Variational Level Set Method and Region Partition Strategy for Multiphase Image Segmentation}

\subsection{Variational Level Set Method for Multiphase Segmentation}

Multiphase image segmentation is using a plurality of label functions to divide an image into some mutually adjacent non-overlapping areas according to the different characteristic. Assuming $f$ is a gray image defined on a rectangular area $\Omega$. By introducing $q$ label functions, the image can be classifed into $p$ areas. The variational level set method for multiphase segmentation is given as follows:

$$
\min _{\phi, u}\left\{E(\phi, u)=\sum_{i=1}^{p} \alpha_{i} \int_{\Omega} Q_{i}\left(u_{i}\right) \chi_{i}(\phi) d x+\sum_{i=1}^{q} \gamma_{i} \int_{\Omega}\left|\nabla H\left(\phi_{i}\right)\right| d x\right\}
$$

Where $\chi_{i}(\phi)$ is the characteristic function and $p \quad q . Q_{i}\left(u_{i}\right)$ denotes the corresponding parameter estimating function for different types of images. $\Omega_{i}, i=1,2, \cdots, p$ satisfies

$$
\Omega=\bigcup_{i=1}^{p} \Omega \quad \Omega \cap \Omega=\varnothing, i \neq j
$$

Characteristic function $\chi_{i}(\phi), i=1,2, \cdots, p$ satisfies

$$
\begin{aligned}
& \chi_{i}(\phi)=\begin{array}{lll}
1 & x & \Omega_{i} \\
0 & x & \Omega_{i}
\end{array}, i=1,2, \cdots, p \\
& { }_{i=1} x_{i}(\phi)=1
\end{aligned}
$$

In order to guarantee that it cannot produce the overlapping or missing segmentation results, (3.1) must meet constraint (3.4). Additionally, in order to let label functions keep the characteristic of signed distance functions in the process of evolution, (3.1) must satisfy (3.5).

$$
\left|\nabla \phi_{i}\right|=1 \quad i=1,2, \cdots, q
$$

\subsection{Segment $n$ regions with $n-1$ label functions}

When using $n-1$ label functions to segment $n$ regions [14-16], we can learn $p=n, q=n-1$. It is

$$
\text { (3.6) } \min _{\phi, u}\left\{E(\phi, u)=\sum_{i=1}^{n} \alpha_{i} \int_{\Omega} Q_{i}\left(u_{i}\right) \chi_{i}(\phi) d x+\sum_{i=1}^{n-1} \gamma_{i} \int_{\Omega} \mid \nabla H\left(\phi_{i}\right) d x\right\}
$$


Then constraint (3.4) becomes to

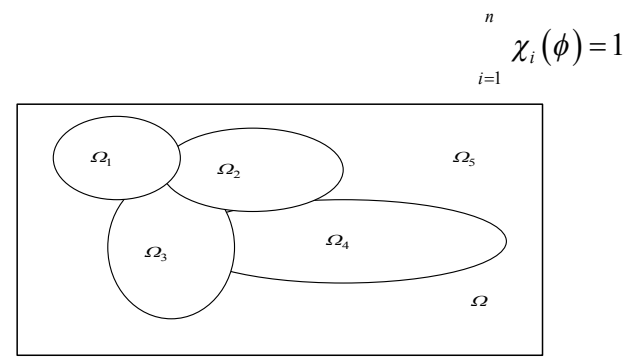

Figure 1: Segmenting five regions with four label functions

Here, we adopted the region partition strategy proposed by Pan et al [16]. The scheme of characteristic functions can be seen in Fig. 1. Here, the corresponding characteristic functions are

$$
\begin{aligned}
& \Omega_{1}: \quad \chi_{1}(\phi)=H\left(\phi_{1}\right)\left(1-H\left(\phi_{0}\right)\right) \\
& \Omega_{2}: \quad \chi_{2}(\phi)=H\left(\phi_{2}\right)\left(1-H\left(\phi_{1}\right)\right)\left(1-H\left(\phi_{0}\right)\right) \\
& \Omega_{3}: \quad \chi_{3}(\phi)=H\left(\phi_{3}\right)\left(1-H\left(\phi_{2}\right)\right)\left(1-H\left(\phi_{1}\right)\right)\left(1-H\left(\phi_{0}\right)\right) \\
& \Omega_{4}: \quad \chi_{4}(\phi)=H\left(\phi_{4}\right)\left(1-H\left(\phi_{3}\right)\right)\left(1-H\left(\phi_{2}\right)\right)\left(1-H\left(\phi_{1}\right)\right)\left(1-H\left(\phi_{0}\right)\right) \\
& \Omega_{5}: \quad \chi_{5}(\phi)=H\left(\phi_{5}\right)\left(1-H\left(\phi_{4}\right)\right)\left(1-H\left(\phi_{3}\right)\right)\left(1-H\left(\phi_{2}\right)\right)\left(1-H\left(\phi_{1}\right)\right)\left(1-H\left(\phi_{0}\right)\right)
\end{aligned}
$$

Then, the general expression of characteristic functions in Fig. 1 is

$$
\chi_{i}(\phi)=H\left(\phi_{i}\right){ }_{j=0}^{i-1}\left(1-H\left(\phi_{j}\right)\right) \quad i=1,2, \cdots, n
$$

here $H\left(\phi_{0}\right)=0$ and $H\left(\phi_{n}\right)=1$. This scheme also satisfy constraint (3.7).

\section{NLMS Model for Multiphase Segmentation and Split Bregman Algorithm}

Here, the multiphase nonlocal Mumford-Shah model is proposed as

$$
\min _{u_{i}: \phi_{i} \in[0,1]}\left\{E(u, \phi)=\sum_{i=1}^{n+1} \alpha_{i} \int_{\Omega}\left(\left(u_{i}-f\right)^{2}+\lambda_{i}\left|\nabla_{N L} u_{i}\right|^{2}\right) \chi_{i}(\phi) d x+\sum_{i=1}^{n} \gamma_{i} \int|\nabla \phi| d x\right\}
$$

We can solve it with alternating optimization approach. First, fixing $\phi_{i}$ for $u_{i}$, we can get

$$
\left(u_{i}-f\right) \chi_{i}(\phi)-\lambda_{i} \nabla_{N L} \cdot\left(\chi_{i}(\phi) \nabla_{N L} u_{i}\right)=0
$$

Thus, the detailed form of $u_{i}$ can be written as

$u_{i}(x)=\frac{f(x) \chi_{i}(\phi)+\lambda_{i}\left(\chi_{i}(\phi(y))+\chi_{i}(\phi(x))\right) u_{i}(y) w(x, y) d y}{\chi_{i}(\phi)+\lambda_{i}\left(\chi_{i}(\phi(y))+\chi_{i}(\phi(x))\right) w(x, y) d y} i=1,2, \cdots, n$

Then, we fix $u_{i}$ to solve $\phi_{i}$. Here, split Bregman algorithm is adopted to solve variable $\phi_{i}$ to improve the computational efficiency. By introducing auxiliary variable $w_{i}$ and Bregman iterative parameter $b_{i}$, (4.1) can be transformed into following form

$$
\underset{w_{i} \phi_{i} \in[0,0,1]}{\operatorname{Min}}\left\{E(\phi, w)=\sum_{i=1}^{n+1} \alpha_{i} \int_{\Omega}\left(\left(u_{i}-f\right)^{2}+\lambda_{i}\left|\nabla_{N L} u_{i}\right|^{2}\right) \chi_{i}(\phi) d x+\sum_{i=1}^{n} \gamma_{i} \int\left|w_{i}\right| d x+\sum_{i=1}^{n} \frac{\theta_{i}}{2} \int\left(w_{i}-\nabla \phi_{i}-b_{i}^{k+1}\right)^{2} d x\right\}
$$

To minimize (4.4), we first fix variable $w_{i}$ for $\phi_{i}$

$$
\nabla \cdot\left(w_{i}^{k}-\nabla \phi_{i}-b_{i}^{k+1}\right)+\sum_{i=1}^{n+1} Q_{i}(u) \frac{\partial \chi_{i}(\phi)}{\partial \phi_{k}}=0
$$


where

$$
\frac{\partial \chi_{i}(\phi)}{\partial \phi_{k}}=\left\{\begin{array}{cc}
\prod_{j=0}^{i-1}\left[1-\phi_{j}\right], & k=i \\
-\prod_{j=0, k \neq i}^{i-1}\left[1-\phi_{j}\right] \phi_{i}, & k \neq i
\end{array}\right.
$$

(4.5) can be solved by one iteration of Gauss-Seidel. Then variable $\phi_{i}$ can be solved efficiently by projection formula

$$
\phi_{i}=\operatorname{Max}\left(\operatorname{Min}\left(\phi_{i}, 1\right), 0\right)
$$

After obtaining ${ }^{\phi_{i}}$, the following soft thresholding equation is used to solve ${ }^{w_{i}}$

$$
w_{i}^{k+1}=\operatorname{Max}\left(\left|\nabla \phi_{i}^{k+1}+b_{i}^{k+1}\right|-\frac{\gamma_{i}}{\theta_{i}}, 0\right) \frac{\nabla \phi_{i}^{k+1}+b_{i}^{k+1}}{\left|\nabla \phi_{i}^{k+1}+b_{i}^{k+1}\right|}
$$

with the convention that $0 / 0=0$.

\section{Numerical Experiments}

In this section, we apply this model on synthetic and real images to verify the performance of the proposed model. We would compare our model with the two-phase Chan-Vese model [17].

In figure 2 and figure 3 , we apply our model on three-phase image segmentation $(n=2)$ and four-phase image segmentation $(n=3)$. It can be seen that the segmentation performance is satisfactory. Here, we list last few rows to better demonstrate the final label functions. It is obvious that those label functions are very close to the binary images $(0$ and 1$)$. Contours gradually approach edges of objects according to different information. The parameters for experiment shown in the first column of figure 2 is $\alpha=1, \lambda=1, \gamma=2000, \theta=5000$. Those for the second column is $\alpha=1, \lambda=1, \gamma=5000, \theta=5000$. And those for figure 3 is $\alpha=1, \lambda=1, \gamma=2000, \theta=8000$.
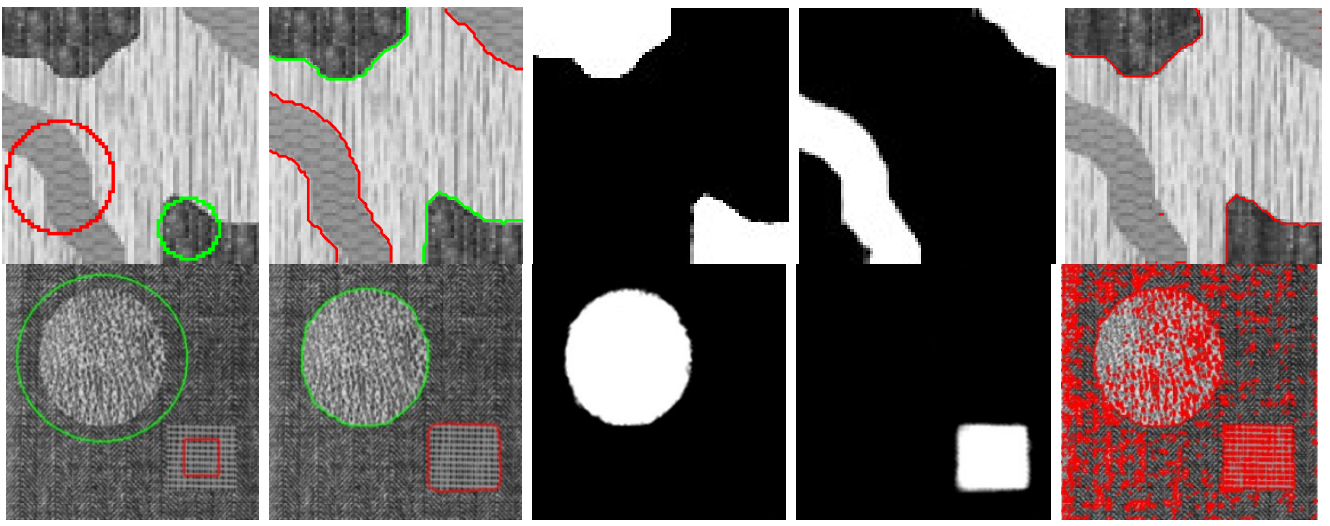

Figure 2 : Three-phase images segmentation. First row: initialization; Second row: segmentation results; Third and fourth row: two label functions $\phi_{1}$ and $\phi_{2}$; Fifth row: segmentation results by two-phase Chan-Vese model 

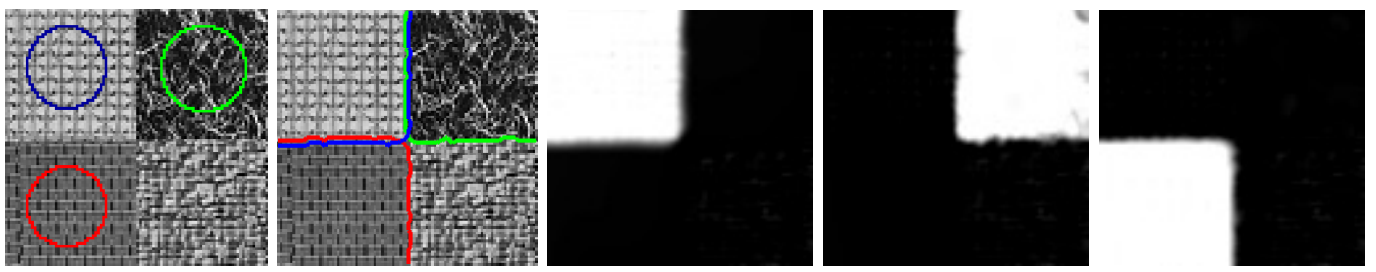

Figure 3: Four-phase images segmentation. First image: initialization; Second image: segmentation results; Third, fourth and fifth images: three label functions $\phi_{1}, \phi_{2}$ and $\phi_{3}$

Figure 4 is the multiphase segmentation results $(n=2)$ for real brain MR image. It shows that proposed model and algorithm can still efficiently segment brain MR images, even though the gray values of edges and area inside brain are very similar. The parameters for this experiment is $\alpha=1, \lambda=1, \gamma=5000, \theta=9000$.

Figure 5 is the multiphase segmentation results $(n=2)$ for $\mathrm{CT}$ images. It can be seen that proposed nonlocal Munford-Shah model and algorithm can segment CT images which contain many tissues efficiently. It is worth mentioning that the region partition strategy cannot produce the overlapping or missing segmentation results as it can automatically satisfy the unique partition condition (i.e., one pixel point can only belong to one segmented region). The parameters for this experiment is $\alpha=1, \lambda=1, \gamma=6000, \theta=1 e 4$.
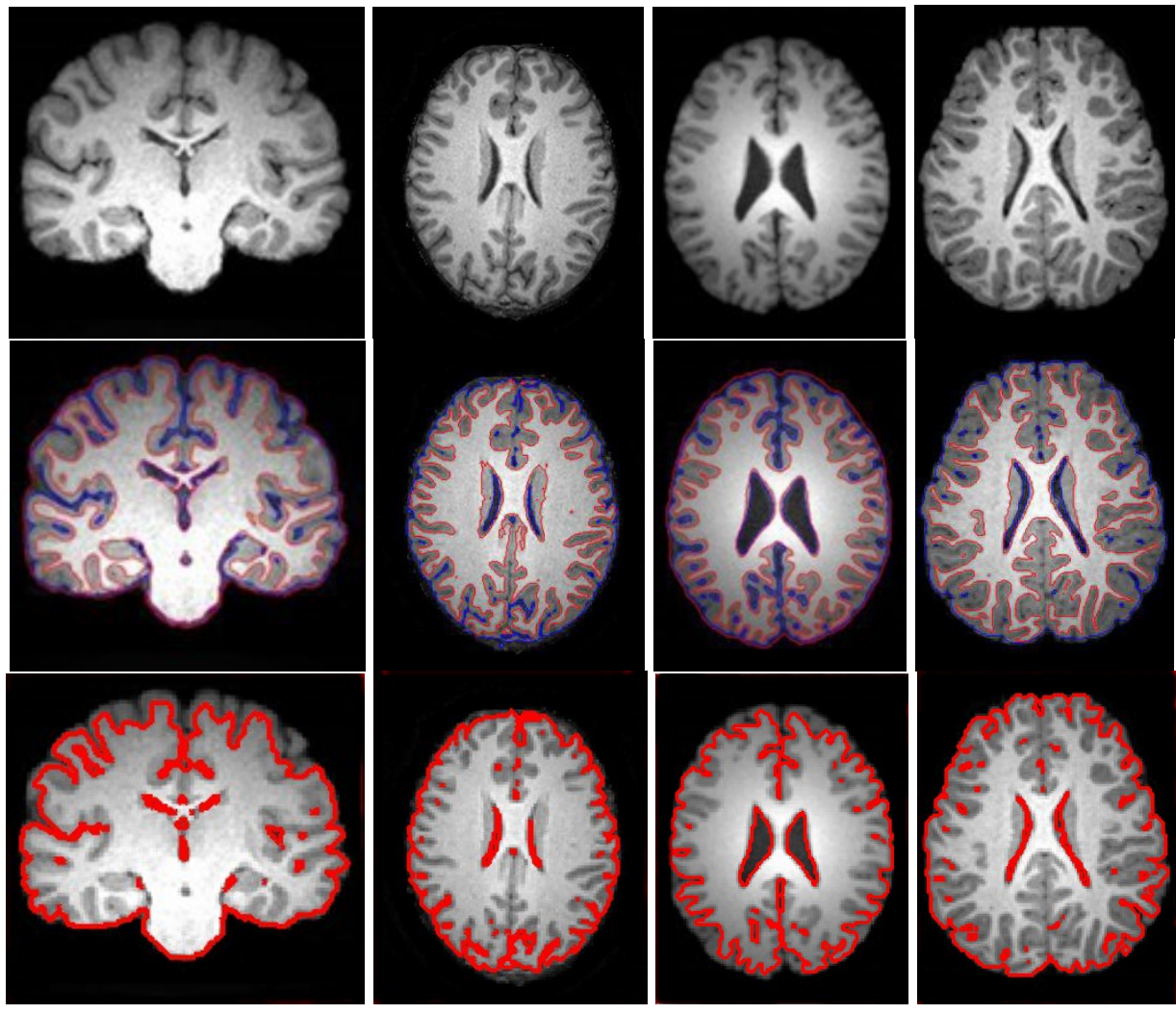

Figure 4 : Multiphase segmentation results for piecewise smooth brain MR images. First column: different original images; Second column: segmentation results; Third column: segmentation results by two-phase Chan-Vese model 


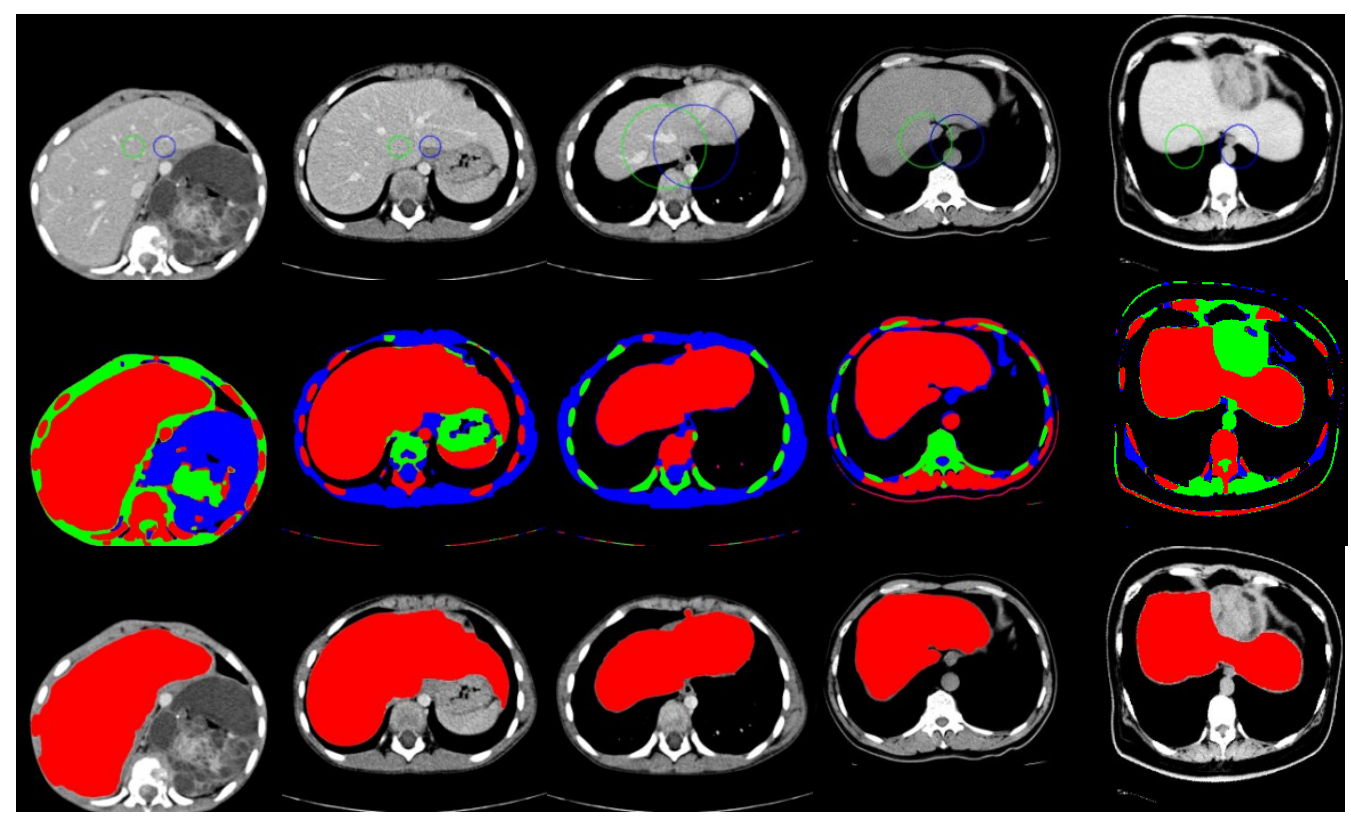

Figure 5 : Multiphase segmentation results for piecewise constant CT images. First column: the contour initialization for different images; Second column: segmentation results; Last column: segmentation of the liver

\section{Conclusion}

In this paper. nonlocal Mumford-Shah model for multiphase image segmentation is proposed. Taking advantage of original Mumford-Shah model, this model combines nonlocal operators with multiple region partition strategy which uses $n-1$ level set functions to segment $n$ regions. So it has ideal performance in multiphase image segmentation. Split Bregman algorithm is designed to increase the computational efficiency. The idea in the paper can be extended to other mathematical problems in image processing.

\section{References}

[1] G. Aubert, P. Kornprobst. Mathematical Problems in Image Processing: Partial Differential Equations and the Calculus of Variations. In: AMS, vol. 147. Springer, Berlin. 1-400(2002)

[2] F. T. Chan, J. Shen. Image processing and analysis: variational, PDE. Wavelet, and Stochastic methods. SIAM, Philadelphia. 1-184(2005)

[3] M. Kass, A. Witriw, D. Terzopoulos. Snakes: Active contour models. International Journal of Computer Vision. 1(4): 321-331(1988)

[4] V. Caselles, R. Kimmel, G. Sapiro. Geodesic active contour. IJCV. 22(1): 61-79(1997)

[5] D. Mumford, J. Shah. Optimal approximations of piecewise smooth functions and associated variational problems. Comm. Pur. Appl. Math. 42(5): 577-685(1989)

[6] T. F. Chan, L. A. Vese. Active Contours Without Edges. IEEE T. Image Process. 10(2): 266277(2001)

[7] S. Osher, N. Paragios. Geometric Level Set Methods in Imaging, Vision, and Graphics. SpringerVerlag, New York 43-58 (2003)

[8] S. Osher, R. Fedkiw. Level Set Methods and Dynamic Implicit Surfaces. Springer-Verlag, New York 1-288(2002) 
[9] L. A. Vese, T. F. Chan. A multiphase level set framework for image segmentation using the Mumford and Shah model. Int. J. Comput. Vision. 50(3): 271-293(2002)

[10] B. Sandberg, T. Chan, L. A. Vese. A Level-set and Gabor Based Active Contour Algorithm for Segmenting Textured Images. UCLA CAM Report 02-39, July(2002)

[11] A. Buades, B. Coll, J. Morel. A Review of Image Denoising Algorithms, with a New One. SIAM MMS. 4(2): 490-530(2005).

[12] G. Gilboa, S. Osher. Nonlocal Operators with Applications to Image Processing. SIAM MMS. 7(3): 1005-1028(2008)

[13] X. Bresson, T. Chan. Nonlocal Unsupervised Variational Image Segmentation Models. UCLA CAM Report 08-67, October(2008)

[14] Li Fang, K. Michael, Zeng Tieyong, Shen Chunlin. A multiphase image segmentation method based on fuzzy region competition. SIAM J. Imaging Sci. 3(3): 277-299(2010)

[15] T. Brox, M. Rousson, R. Deriche, J. Weickert. Colour, texture, and motion in level set based segmentation and tracking. Image Vision Comput. 28(3): 376-390(2010)

[16]Z. K. Pan, H. Li, W.B. Wei, Z. B. Guo, C. F. Zhang. A Variational Level Set Method of Multiphase Segmentation for 3D Images. Chin. Journal of Computers. 32(12): 2464-2474(2009) (In Chinese)

[17] J.M. Duan, Z. K. Pan, X.F. Yin, W.B. Wei, G.D. Wang. Some fast projection methods based on Chan-Vese model for image segmentation. Eurasip J.Image.Vide. Process. 2014(1): 1-16(2014) 